\title{
WWP1 germline variants are associated with normocephalic autism spectrum disorder
}

\author{
Giuseppe Novelli i $\mathbb{1}^{1,2,3}$, Antonio Novellii, Paola Borgiani ${ }^{1}$, Dario Cocciadiferro ${ }^{4}$, Michela Biancolella ${ }^{5}$, Emanuele Agolini ${ }^{4}$, \\ Marco Pietrosanto ${ }^{5}$, Rosario Casalone ${ }^{6}$, Manuela Helmer-Citterich ${ }^{5}$, Emiliano Giardina ${ }^{1,7}$, Suresh K. Jain ${ }^{8}$, \\ Wenyi Wei $\mathbb{1}^{9,10}$, Charis Eng (10) ${ }^{11,12,13,14}$ and Pier Paolo Pandolfit ${ }^{15,16}$
}

Dear Editor,

Autism spectrum disorder (ASD, MIM: 209850) is a group of common but heterogeneous neurodevelopmental disorders with a prevalence of 4-10 per 10,000 individuals $^{1,2}$. About $5 \%$ of ASD cases are caused by single-gene variants in FMR1 (MIM: 309550), MECP2 (MIM: 300005), or SHANK3 (MIM: 606230); 10\% by copy number variants $(\mathrm{CNVs})^{2}$, while the majority is attributed to polygenic inheritance of common variants ${ }^{3}$. In addition, germline PTEN mutations have been identified in $2-5 \%$ of all ASD patients and $\sim 10 \%$ of macrocephalic $\mathrm{ASD}^{4}$. Recently, Lee et al. ${ }^{5}$ identified germline variants within the E3 ubiquitin ligase WWP1 (MIM: 602307) gene in PTEN mutation negative individuals with neoplastic phenotypes found in PHTS (MIM: 158350).

To establish whether WWP1 could play a role in ASD and neurodevelopment disorders, we analyzed 198 unrelated individuals mainly referred for syndromic or nonsyndromic developmental delay and/or ASD of unknown genetic etiology. All individuals were clinically diagnosed with ASD on the basis of the fourth edition of the Diagnostic and Statistical Manual of Mental Disorders (DSMIV). Whole-exome sequencing, validated by Sanger sequencing, identified eight different heterozygous germline mutations (one recurrent in three unrelated patients) of the WWP1 gene in 10 of 198 unrelated probands via WES (Table 1). None of the variant positive probands had macrocephaly. In two cases, parental origin could not be investigated, therefore, a de novo origin of

\footnotetext{
Correspondence: Giuseppe Novelli (novelli@med.uniroma2.it) or

Pier Paolo Pandolfi (pierpaolo.pandolfiderinaldis@unito.it)

'Department of Biomedicine and Prevention, Tor Vergata University of Rome, 00133 Rome, Italy

${ }^{2}$ IRCCS Neuromed, Pozzilli (IS), Italy

Full list of author information is available at the end of the article
}

the mutation, cannot be ruled out. For each patient (6 males and 4 females; ages 3-26), the clinical data have been reassessed. None of the probands had germline PTEN mutations or other mutations in genes (FMR1, SHANK3, MECP2, CDK19) associated with ASD/intellectual disability (ID). We independently confirmed that WWP1 variation does not act as a modifier for ASD phenotypes in PHTS with none of $\sim 600$ mainly American PTEN mutation positive research associated with the WWP1 locus. Similarly, routine chromosome studies and FRAXA locus were normal. GnomAD database analysis revealed that the identified WWP1 variants with the exception of R389S, R893H, and M728L (never detected), existed with a cumulative frequency of 0.00085 in ethnically matched populations (EUR), indicating that they are very rare variants. Specifically, WWP1 germline variants occurred in 10/396 alleles (allelic freq. $=0.0252$ ) from the 198 unrelated individuals with ASD/ID (Table 1) which is a highly significant difference from European population frequencies from GnomAD $(p<0.00001$; OR $=30.6$ with 95\% CI 16.27 and 57.59). We therefore extended the study to a cohort of 1158 individuals from the Italian general population to establish the frequency of $W W P 1$ variants in this Italian cohort. We detected three WWP1 rare variants (c.1118G $>A, \quad$-Arg373Gln; c.1486G $>C$, p. Glu496Gln; c.2234A $>$ G, p.Asn745S) (3/2316 alleles: allelic freq. $=0.00129)$. Notably, WWP1 variants were again shown to be over-represented in the ASD/ID series, even when compared with the Italian cohort examined $(p<$ $0.00001 ; \mathrm{OR}=19.93$ with $95 \% \mathrm{CI} 5.47$ and 72.90 ). The variants are found in all functional domains of the protein (the catalytic C-terminal HECT domain; the N-terminal C2 domain and WW domains) with an overrepresentation in the HECT domain (4/8). To predict the potential impact of the identified variants on the 
Table 1 Summary of variations in ASD patients carrying WWP1 mutations.

\begin{tabular}{llllllllll}
\hline Patient ID & Sex & Exon & Position (Hg19) & Nucleotide & Amino acid & Domain & GnomAd $^{\text {a }}$ & dbSNP & Transmission \\
\hline GM4277 & F & Int 7 & 87414243 & c.540-5T>C & & NA & 0.0027 & rs187132881 & Mother \\
GM3474 & M & 11 & 87439881 & c.1167A>C & p.Arg389Ser & WW1 & 0 & NA & NA \\
A020 & M & 14 & 87443954 & c.1583G>A & p.Arg528His & WW4 & 0.000008 & rs554041348 & Father \\
GM6802 & F & 20 & 87460703 & c.2234A>G & p.Asn745Ser & HECT & 0.00003 & rs 148651938 & Mother \\
GM8105 & M & 20 & 87460703 & c.2234A>G & p.Asn745Ser & HECT & 0.00003 & rs148651938 & Father \\
GM-1HSL & M & 20 & 87460703 & c.2234A>G & p.Asn745Ser & HECT & 0.00003 & rs 148651938 & NA \\
GM4098 & F & 20 & 87460645 & c.2176G>A & p.Val726lle & HECT & 0.000023 & rs 144129917 & Mother \\
GM8302 & F & 25 & 87479031 & c.2678G>A & p.Arg893His & HECT & 0 & rs 755897749 & Father \\
A036 & M & 20 & 87460651 & c.2182A>T & p.Met728Leu & HECT & 0 & NA & Father \\
A069 & M & 5 & 87393781 & c.257G>A & p.Arg86His & C2 & 0.000023 & rs371650373 & Mother \\
\hline
\end{tabular}

${ }^{\mathrm{a}} \mathrm{EUR}$.

protein we used different tools (PolyPhen2, Mutation Taster, SIFT, MetaLR_pred, and MetaSVM_pred). The recurrent $\mathrm{N} 745 \mathrm{~S}$ variant has been previously reported by Lee et al. $^{5}:$ it is in the HECT domain and is expected to decrease its binding to the N-terminal domain. Analogously, $\mathrm{R} 86 \mathrm{H}$ (C2 domain) was also described by Lee et al. ${ }^{5}$. This variant is functionally relevant since it induces a gain-of-function effect in triggering PTEN polyubiquitination $^{5}$. With regards to the other five coding variants observed in our ASD cases, one is predicted by in silico analysis to be deleterious $(\mathrm{R} 528 \mathrm{H})$, while the others gave conflicting results.

Our results suggest that germline WWP1 variants identified in ASD/ID/NDDs may contribute to the pathogenesis of ASD/ID/NDDs. In addition, since the enzymatic activity of WWP1 can be inhibited by the natural compound, indole-3-carbinol ${ }^{6}$, our study identifies a possible therapeutic target for individuals with ASD/ID/NDDS.

\section{Web resources}

GnomAD, https:/gnomad.broadinstitute.org/

PolyPhen2, http://genetics.bwh.harvard.edu/pph2/

Mutation Taster, http://www.mutationtaster.org/

SIFT, https://sift.bii.a-star.edu.sg/

OMIM, https://OMIM.org/.

\section{Acknowledgements}

We are grateful to the participating families and II Ponte del Sorriso Onlus for its support. PPP was supported by the NCl grant R35CA197529 and in part by the PTEN Research Foundation.

\section{Author details}

${ }^{1}$ Department of Biomedicine and Prevention, Tor Vergata University of Rome, 00133 Rome, Italy. ${ }^{2}$ IRCCS Neuromed, Pozzilli (IS), Italy. ${ }^{3}$ Department of

Pharmacology, School of Medicine, University of Nevada, Reno, NV 89557, USA. ${ }^{4}$ Laboratory of Medical Genetics, Bambino Gesù Children's Hospital, IRCCS, Rome, Italy. ${ }^{5}$ Department of Biology, Tor Vergata University of Rome, 00133 Rome, Italy. ${ }^{6}$ Cytogenetics and Medical Genetics Laboratory, Ospedale di Circolo, ASST Sette Laghi, Varese, Italy. ${ }^{7}$ Molecular Genetics Laboratory UILDM,
Santa Lucia Foundation, 00142 Rome, Italy. ${ }^{8}$ Intonation Research Laboratories Pvt. Ltd, Hyderabad, Telangana 500076, India. ${ }^{9}$ Cancer Research Institute, Beth Israel Deaconess Cancer Center, Harvard Medical School, Boston, MA 02215, USA. ${ }^{10}$ Department of Pathology, Beth Israel Deaconess Medical Center, Harvard Medical School, Boston, MA 02215, USA. ${ }^{11}$ Genomic Medicine Institute, Lerner Research Institute, Cleveland Clinic, Cleveland, OH 44195, USA. ${ }^{12}$ Taussig Cancer Institute, Cleveland Clinic, Cleveland, OH 44195, USA. ${ }^{13}$ Department of Genetics and Genome Sciences, Case Western Reserve University School of Medicine, Cleveland, OH 44106, USA. ${ }^{14}$ Germline High Risk Cancer Focus Group, Case Comprehensive Cancer Center, Case Western Reserve University, Cleveland, $\mathrm{OH}$ 44106, USA. ${ }^{15} \mathrm{MBC}$, Department of Molecular Biotechnology and Health Sciences, University of Turin, Turin, TO 10126, Italy. ${ }^{16} \mathrm{DRI}$, Renown Health, Nevada System of Higher Education, Reno, NV 89512, USA

\section{Conflict of interest}

The authors declare that they have no conflict of interest.

\section{Ethical declarations}

The study was conducted in agreement with the principles of the Declaration of Helsinki. Informed written consent was obtained from each patients. As regards the participation of children in the research, consent and authorization were signed by the parents in accordance with the rules laid down by the Ethics Committee of the Bambino Gesù Hospital in Rome (HYPERLINK "https:// urldefense.proofpoint.com/v2/url?u=http-3A__www.ospedalebambinogesu. it_en_home\&d=DwMFaQ\&c=vh6FgFnduejNhPPD0fl_yRaSfZy8CWbWnlf4XJhSqx8\&r=H8EiHZYdOfzgj3SnkNr1OWfOZuk7ldFteVpx6F9BizvoZAKx_zllbLuDzKXrCwF8\&m=0dvSb4bLNoeGzXhLeNXyRGhxjEoUL6Qd_oj7reRTsMg\&s=FMPyM3gTbUpOHGox37ytL4D0gGUI3gocQICNX9p-1IE\&e=" MailScanner ha rilevato un possibile tentativo di frode proveniente da "urldefense.proofpoint.com" http://www.ospedalebambinogesu.it/en/home).

\section{Publisher's note}

Springer Nature remains neutral with regard to jurisdictional claims in published maps and institutional affiliations.

Received: 18 May 2020 Revised: 26 May 2020 Accepted: 3 June 2020 Published online: 23 July 2020

\section{References}

1. Charles, J. M. Autism spectrum disorders: an introduction and review of prevalence data. J. S. C. Med. Assoc. 102, 267-270 (2006).

2. Stefansson, $\mathrm{H}$. et al. CNVs conferring risk of autism or schizophrenia affect cognition in controls. Nature 505, 361-366 (2014). 
3. Clarke, T. K. et al. Common polygenic risk for autism spectrum disorder (ASD) is associated with cognitive ability in the general population. Mol. Psychiatry 21, 419-425 (2016)

4. Yehia, L., Ngeow, J. \& Eng, C. PTEN-opathies: from biological insights to evidence-based precision medicine. J. Clin. Invest 129, 452-464 (2019).
5. Lee, Y. R. et al. WWP1 gain-of-function inactivates PTEN to drive cancer predisposition. N. Eng. J. Med. 382, 2103-2116 (2020).

6. Lee, Y. R. et al. Reactivation of PTEN tumor suppressor for cancer treatment through inhibition of a MYC-WWP1 inhibitory pathway. Science 364, eaau0159 (2019). 